\title{
Erratum to: High-accuracy coherent optical frequency transfer over a doubled 642-km fiber link
}

D. Calonico - E. K. Bertacco - C. E. Calosso - C. Clivati •

G. A. Costanzo $\cdot$ M. Frittelli $\cdot$ A. Godone $\cdot$ A. Mura $\cdot$

N. Poli · D. V. Sutyrin - G. M. Tino $\cdot$ M. E. Zucco $\cdot$ F. Levi

Published online: 7 December 2014

(C) Springer-Verlag Berlin Heidelberg 2014

Erratum to: Appl. Phys. B (2014) 117:979-986

DOI 10.1007/s00340-014-5917-8

Unfortunately, the mid-initial of the eleventh author was not published in the original publication of the article. The correct name of the author should be: "G. M. Tino".

The online version of the original article can be found under doi:10.1007/s00340-014-5917-8.

D. Calonico - E. K. Bertacco - C. E. Calosso - C. Clivati $(\bowtie)$.

G. A. Costanzo - M. Frittelli - A. Godone - A. Mura ·

M. E. Zucco · F. Levi

Istituto Nazionale di Ricerca Metrologica INRIM, Strada delle

Cacce 91, 10135 Turin, Italy

e-mail: c.clivati@inrim.it

G. A. Costanzo

Politecnico di Torino, Corso Duca degli Abruzzi 24,

10129 Turin, Italy

N. Poli · D. V. Sutyrin · G. M. Tino

Universitàdi Firenze, LENS and INFN, Via Sansone 1,

Sesto Fiorentino, FI, Italy 